\title{
Aptitud Física y su Relación con Rasgos Depresivos en Personas Adultas Mayores que Realizan Actividad Física
}

\author{
Physical Fitness and its Relationship to Depressive Traits in Older Adults Undertaking \\ Physical Activity
}

\author{
Mynor Rodríguez-Hernández ${ }^{1}$, Felipe Araya Ramírez, Pedro Ureña \\ Bonilla $^{2}$, Danielle D. Wadsworth ${ }^{1}$ y Luis Solano Mora ${ }^{2}$ \\ ${ }^{1}$ School of Kinesiology, Auburn University, Alabama, USA. \\ ${ }^{2}$ Escuela de Ciencias del Movimiento Humano y Calidad de Vida, \\ Universidad Nacional, Costa Rica \\ mgr0018@tigermail.auburn.edu
}

\begin{abstract}
Resumen
El propósito de esta investigación fue determinar la relación entre la aptitud física y los niveles depresivos en las personas adultas mayores que participan en programas de actividad física en el área de San Ramón, Alajuela. Un total de 138 personas mayores con edades entre los 60 y 86 años (67.94 \pm 5.26 años), fueron medidas en los componentes de la aptitud física mediante la prueba "Senior Fitness Test" (SFT) y los niveles de depresión mediante la Escala de Depresión Geriátrica de "Yesavage" (GDS, "Geriatric Depression Scale" por sus siglas en Inglés). Los resultados sugieren que el 97,8 \% de las personas analizadas se ubicó dentro de los parámetros normales y excelentes de aptitud física, mientras tanto, el 86,2 \% se encontró en la etapa normal de la depresión. Por su parte, la depresión geriátrica correlacionó negativamente con la aptitud física en las variables: fuerza muscular y resistencia corporal de la parte baja del cuerpo, resistencia de la fuerza muscular del brazo, la agilidad y equilibrio dinámico, y flexibilidad parte baja del cuerpo $(p<0,05)$. Se concluye que a mayor nivel de aptitud física menor son los rasgos depresivos en personas adultas mayores.
\end{abstract}

Palabras Claves: Depresión, persona adulta mayor, envejecimiento, aptitud física, actividad física.

\begin{abstract}
The purpose of this study was to determine the relationship between physical fitness and levels of depression in older persons involved in physical activity programs in San Ramon, Alajuela. A total of 138 older adults aged 60 to 86 years $(67.94 \pm 5.26)$ were assessed on the physical fitness using the SFT and levels of depression were measured using the GDS. Results suggest that about $97.8 \%$ of persons tested were within normal parameters and excellent on their physical fitness. Additionally, $86.2 \%$ of the cohort was found in a normal stage of depression. In addition, geriatric depression was negatively correlated with physical fitness on variables such as lower body muscle strength and endurance, arm muscle strength endurance, physical agility and dynamic balance, and lower body flexibility $(p<0.05)$. It is concluded that higher levels of physical fitness are associated with lower depression status in older adults.
\end{abstract}

Key Words: Depression, older adults, aging, physical fitness, physical activity. 


\section{Introducción}

La depresión es de uno los factores de mayor afectación sobre la calidad de vida de las personas adultas mayores y está asociado a altos niveles de mortalidad y morbilidad (Strawbridge, Deleger, Roberts, y Kaplan, 2002). De acuerdo con datos suministrados por Wada y otros (2005), la depresión en las personas adultas mayores no solo es una gran amenaza para la salud mental, sino también un motivo de preocupación en cualquier campo de intervención en esta población. Por consiguiente el envejecimiento exitoso, depende en gran medida de la atención integral y de la intervención oportuna de estas situaciones que afectan el buen vivir de una persona adulta mayor. En el estudio de Wada y otros (2005), se determinó la existencia de estados depresivos entre el 17 al $32 \%$ de las personas evaluadas, además, se observó que esta patología se asocia fuertemente con una baja calidad de vida y con una reducida capacidad para realizar actividades de la vida diaria. Por su parte Menchetti, Cevenini, De Ronchi, Quartesan, y Berardi (2006) indican que la depresión es un factor de riesgo importante de morbi-mortalidad y que afecta de manera negativa, independientemente de otras patologías físicas y somáticas.

La depresión en las personas adultas mayores es un trastorno psicológico que se relaciona con múltiples situaciones que afectan la salud y la calidad de vida. De acuerdo con Orfila y otros (2006), la depresión influye directamente sobre la capacidad social de la persona, el bienestar psicológico y sobre su funcionalidad física, aspectos directamente relacionados con el aumento de la morbilidad y mortalidad de la población de personas adultas mayores.

Datos de la Organización Panamericana de la Salud (OPS) indican que en el 2004, alrededor de 121 millones de personas en el mundo se vieron afectadas por la depresión y que entre el 15 al $25 \%$ de la población mundial sufrirá de depresión al menos una vez en la vida. Según Runcan, Hategan, Barbat y Alexiu (2010) la depresión llegará a ocupar en el 2020 el segundo lugar en las enfermedades de mayor incidencia en la población, después de la hipertensión arterial. En este estudio hay factores descritos como disparadores de la depresión; por ejemplo: el impacto negativo del retiro, el cual hace que incremente el riesgo de depresión en un $15 \%$; la pérdida de un ser querido genera esta patología en el $90 \%$ de los casos, el $90 \%$ de las personas visualizan la pobreza como un factor que puede desarrollar depresión y entre el 32 al $40 \%$ indican que la soledad es un factor detonante de esta patología. Además, se menciona que la depresión afecta las relaciones sociales, la capacidad para realizar las actividades de la vida diaria y la calidad de vida. En otro estudio, Heun y Hein (2005) mencionan algunos factores de riesgo asociados a la depresión, tales como: el sexo femenino, el bajo nivel educativo, la presencia de enfermedades físicas, las limitaciones funcionales, una red social pequeña y un bajo locus de control externo.

Sin embargo, uno de los factores que más afecta a dicha población es el bajo nivel de actividad física el cual se relaciona directamente con niveles depresivos importantes. Así, por ejemplo, Wada y otros (2005) reportaron niveles de depresión del 33,8 \% en Indonesia, 17,2 $\%$ en Vietnam y 30,3 \% en Japón. En Estados Unidos, Whooley, Stone y Soghikian (2000) reportaron un $14 \%$ de depresión en personas adultas mayores, y en Estonia un 40,2\% de personas sufría esta enfermedad (Saks, Tiit, Kaarik y Jaanson, 2002). Mientras tanto, en Costa Rica, según datos de la OPS en el 2004, se encontró que el $38 \%$ de las personas adultas mayores estaban en riesgo de depresión y el $10 \%$ ya sufrían depresión. Estos datos indican, además, que las personas con mayor nivel de actividad física diaria son las que tienen más 
Rodríguez Hernández, M., Araya Ramírez, F., Ureña Bonilla, P., Wadsworth1, D.D. y Solano Mora, L. probabilidad de tener un estado de ánimo normal. Por su parte, Cáceres (2004) encontró que el $52 \%$ de las personas de Costa Rica presentaban un estado depresivo normal, el 37,9\% se encontraban en riesgo de depresión y el 10,1\% estaban en estado depresivo.

El deterioro funcional de la persona adulta mayor es un proceso biológico normal que disminuye la aptitud física y da paso a problemas emocionales conducentes a estados depresivos relacionados con la imposibilidad de realizar normalmente las actividades de la vida diaria. Sin embargo, la actividad física regular ha demostrado ser un elemento indispensable para el manejo de la depresión en esta población. En su investigación, Mather, Rodríguez, Guthrie, McHarg, Reid y McMurdo (2002) encontraron que luego de 10 semanas de participación en un programa de ejercicio, los niveles depresivos de las personas involucradas disminuyeron significativamente, y que aún pequeños periodos de actividad física resultaron beneficiosos para las personas con niveles depresivos altos.

En un estudio de seguimiento con casi 7000 mil personas adultas mayores, Strawbridge y otros (2002) encontraron una relación negativa entre la actividad física y la depresión. Además, determinaron que la actividad física tiene un efecto protector contra la depresión no solo inmediato sino que ayuda a que las personas tengan menos posibilidades de desarrollar esta enfermedad a futuro. Desde el punto de vista fisiológico, la producción de una serie de sustancias como la epinefrina, norepinefrina, endorfinas y otras sustancias liberadas como resultado de la actividad física junto el aumento de la irrigación sanguínea, producen efectos positivos en la sensación de bienestar general disminuyendo las posibilidades de generar estados depresivos y aumentando la calidad de vida. Además, Strawbridge y otros (2002) y Mura y Carta (2013) indican que la autoestima que genera la actividad física y la mejoría en la aptitud física, tiene efecto protector en contra de la depresión, y se ha reportado que a mayores niveles de aptitud física, menores son los rasgos depresivos presentes.

Así mismo, Unsar y Sut (2010) reportaron que la depresión tiene relación directamente con el estado de salud de las personas adultas mayores y que a mayor depresión se da una menor calidad de vida. El riesgo de padecer de depresiones incrementa en aquellas personas con enfermedades crónicas degenerativas. En su estudio realizado con pacientes hospitalizados, se encontró que el $64 \%$ de ellos padecía de depresión y se indicó que el apoyo dirigido a atender las necesidades psico-sociales y a la mejora de la de aptitud física ayuda significativamente a mejorar la calidad de vida de los pacientes con edad avanzada y con estados depresivos presentes.

Se ha investigado la relación existente entre el ejercicio físico y la depresión en personas adultas mayores, sin embargo la relación entre los niveles de la aptitud física y la depresión no están completamente establecidos. Por lo tanto, el propósito de este estudio fue determinar la relación existente entre los niveles de aptitud física y los rasgos depresivos en personas adultas mayores que realizan un programa estructurado de actividad física en el área de San Ramón, Alajuela. 


\section{Metodología}

\section{Participantes.}

Se evaluó un total de 138 personas adultas mayores, con edades entre 60 y 86 años con una edad promedio de $67.94 \pm 5.26$ años. De estos, 120 fueron mujeres y 18 hombres. Estas personas fueron escogidos de los programas de actividad física institucionalizados del área de San Ramón. La experiencia previa realizando actividad física a través de un programa organizado no fue un factor considerado como inclusión o exclusión de los participantes. Los participantes luego de explicarse lo referente a la investigación accedieron a firmar el debido consentimiento informado aprobado por la Comité Ético Científico de la Vicerrectoría de Investigación de la Universidad de Costa Rica.

\section{Instrumentos.}

Se utilizó la Escala de Depresión Geriátrica para medir los niveles depresivos en personas adultas mayores. El cuestionario consiste de preguntas en la que los participantes deben responder "sí" o "no" con respecto a cómo se sintieron en la última semana. La sensibilidad del GDS es del $92 \%$ y la especificidad es del $89 \%$ (Kurlowicz, 2007). Las personas que obtienen menos de 11 puntos en esta escala se consideran con un nivel normal depresivo, las personas con puntajes entre 11 y 14 puntos en estado depresivo I y $>14$ puntos en estado depresivo II (Kurlowicz, 2007).

Además, para medir la aptitud física funcional se utilizó el SFT, el cual es una prueba física estandarizada que evalúa la capacidad para realizar actividades de la vida diaria y de manera independiente en personas adultas mayores. El SFT cuenta con 7 pruebas que evalúan los componentes de la Aptitud Física de las personas adultas mayores. Estas pruebas son: 1. Prueba de levantarse de un silla, "Chair Stand test", (correlación $r=.78$ hombres y $r=.71$ mujeres, confiabilidad $\alpha 0.86$ hombres y $\alpha 0.92$ mujeres); 2 . Prueba de curl de bíceps, "Arm Curl test", (correlación de $r=.84$ hombres y $r=.79$ mujeres, confiabilidad $\alpha 0,81$ hombres y $\alpha$ 0,80 mujeres); 3. Prueba de caminata de 6 minutos, "6 Minutes Walk", (correlación de $r=.82$ hombres y $r=.71$ mujeres, confiabilidad $\alpha 0.97$ para hombres y $\alpha 0.91$ para mujeres); 4. Prueba de sentarse en una silla y alcanzar, "Chair Sit \& Reach test", (correlación $r=.81$ mujeres y de $r=.76$ hombres, confiabilidad $\alpha 0.96$ mujeres y de $\alpha 0.92$ hombres); 5 . Prueba de rascarse la espalda, "Back Scratch test", (confiabilidad $\alpha 0.96$ hombres y a 0.92 mujeres); 6 . Prueba de levantarse, caminar describiendo un 8 y sentarse, "8-Foot Up -and- Go test", ( $r=$ .81 y $\alpha 0.98$ hombres y $\alpha 0.90$ mujeres); y 7 . Indice de masa corporal (IMC), el cual se obtiene dividiendo el peso en kilogramos entre la estatura al cuadrado.

\section{Procedimiento.}

Se recolectó la información a través de la Escala de Depresión Geriátrica (GDS, "Geriatric Depression Scale" por sus siglas en Inglés), la cual puede usarse con adultos mayores con buena salud, con enfermedad conocida y en aquellos con deterioro cognitivo de leve a moderado. Seguidamente, se aplicó el SFT para medir la aptitud física al realizar las actividades de la vida diaria, de modo seguro, con independencia y sin fatiga (Rikli y Jones, 2001). Las mediciones del SFT se llevaron a cabo en dos ocasiones diferentes. La primera medición se realizó para que las personas se familiarizaran con cada una de las pruebas. Luego, en la segunda medición, se recolectaron los datos de cada una de las pruebas que fueron utilizados para el análisis. Asimismo, las personas adultas mayores se agruparon de 

acuerdo a su edad en cuatro subgrupos: a) 60-64 años, b) 65-69 años, c) 70-74 años y d) 75 años y más.

\section{Análisis estadístico.}

Se utilizó la estadística descriptiva (promedios y desviaciones estándar) para cada variable dependiente, así como correlaciones de "Pearson". Para determinar la normalidad de los datos se utilizó la prueba de "Kolgomorov Smirnov test". Además, se utilizó el análisis de varianza (ANOVA) de una vía para determinar diferencias entre los grupos por edad. En los casos en que la distribución no fue normal se utilizó la prueba Kruskal-Wallis. Se trabajó con un nivel de significancia de $p<0.05$ y los análisis fueron hechos con el paquete estadístico para las ciencias sociales "SPSS" versión 22.0.

\section{Resultados}

Las características descriptivas de las personas adultas mayores por rango de edad se presentan en la tabla 1.

Tabla 1.

Características descriptivas de las personas adultas mayores por rango de edad

\begin{tabular}{llcccc}
\hline Rango edad & & Edad & $\begin{array}{c}\text { PESO } \\
\text { kg }\end{array}$ & $\begin{array}{c}\text { TALLA } \\
\text { m }\end{array}$ & \multirow{2}{*}{ IMC } \\
\hline \multirow{3}{*}{ 60-64 años } & $n$ & 39 & 39 & 39 & 39 \\
& $S D$ & 1.353 & 11.0794 & 0.05792 & 3.3909 \\
& Promedio & 61.9 & 67.033 & 1.5581 & 27.549 \\
$\mathbf{6 5 - 6 9}$ años & $n$ & 48 & 48 & 48 & 48 \\
& $S D$ & 1.57 & 11.0443 & 0.06657 & 4.3663 \\
& Promedio & 67.04 & 65.017 & 1.5425 & 27.198 \\
$70-74$ años & $n$ & 35 & 35 & 35 & 35 \\
& $S D$ & 1.336 & 10.1813 & 0.08225 & 3.9975 \\
& Promedio & 71.46 & 66.026 & 1.5607 & 27.209 \\
75 años y más & $n$ & 16 & 16 & 16 & 16 \\
& $S D$ & 2.938 & 10.0502 & 0.11435 & 3.2573 \\
& Promedio & 77.69 & 64.337 & 1.5622 & 26.394 \\
Total & $N$ & 138 & 138 & 138 & 138 \\
& $S D$ & 5.261 & 10.6578 & 0.07505 & 3.8688 \\
& Promedio & 67.94 & 65.764 & 1.5538 & 27.207 \\
\hline
\end{tabular}

Los resultados de las pruebas del SFT se presentan en la figura 1. Las prueba de flexión de brazo, caminar 6 minutos, flexibilidad de tronco, flexibilidad de hombros y la prueba de levantarse, caminar formando la figura de un 8 y sentarse se ubicaron entre los percentiles 25 y el 75, es decir, un estado físico funcional normal. En la prueba de sentarse y levantarse de una silla se obtuvo un valor percentil promedio de 80 , lo cual se ubica en un estado físico excelente. El resultado promedio del IMC fue $27,2 \pm 3,9 \mathrm{~kg} / \mathrm{m}^{2}$, lo cual muestra un nivel de sobrepeso en el 55,1\% de los casos. No se reportaron diferencias significativas en ninguna de las variables del SFT según los rangos de edad. 
Rodríguez Hernández, M., Araya Ramírez, F., Ureña Bonilla, P., Wadsworth1, D.D. y Solano Mora, L.

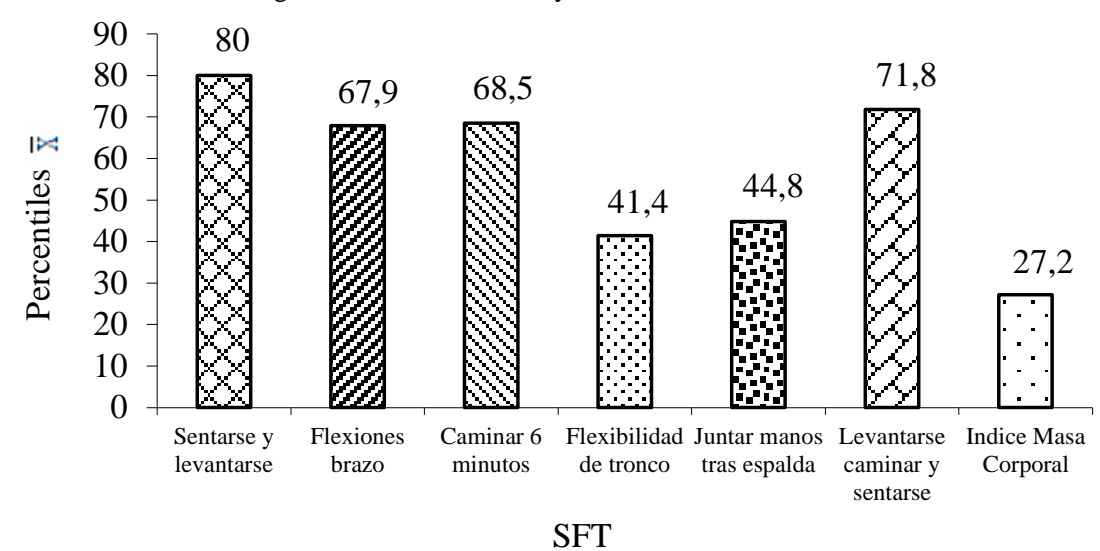

Figura 1. Valores percentiles promedio obtenidos en el Senior Fitness Test de manera general, en las personas adultas mayores que asisten a programas institucionalizados

Los resultados de las pruebas del SFT se presentan en la figura 1. Las prueba de flexión de brazo, caminar 6 minutos, flexibilidad de tronco, flexibilidad de hombros y la prueba de levantarse, caminar formando la figura de un 8 y sentarse se ubicaron entre los percentiles 25 y el 75, es decir, un estado físico funcional normal. En la prueba de sentarse y levantarse de una silla se obtuvo un valor percentil promedio de 80 , lo cual se ubica en un estado físico excelente. El resultado promedio del IMC fue $27,2 \pm 3,9 \mathrm{~kg} / \mathrm{m}^{2}$, lo cual muestra un nivel de sobrepeso en el 55,1\% de los casos. No se reportaron diferencias significativas en ninguna de las variables del SFT según los rangos de edad.

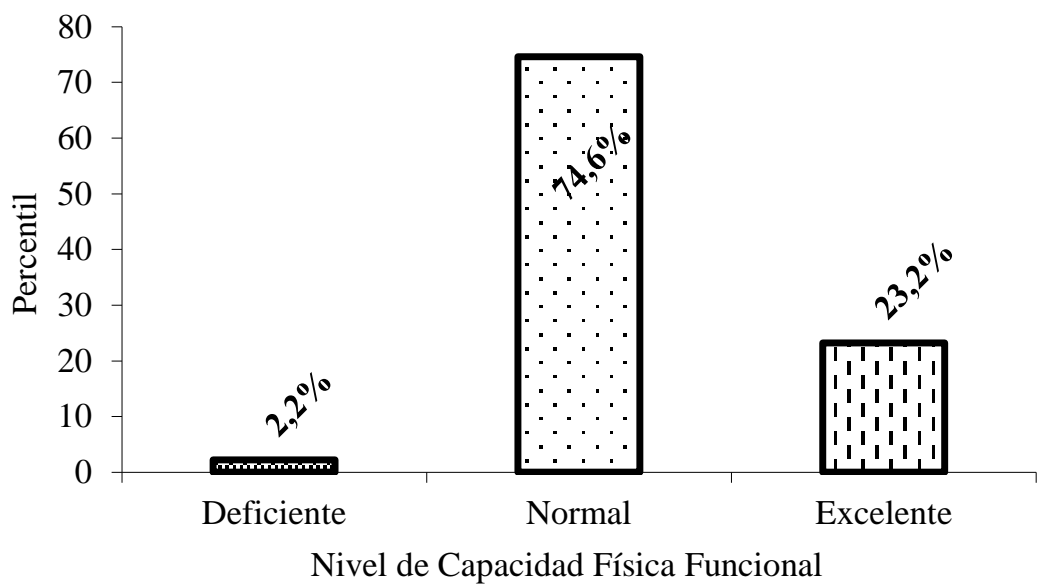

Figura 2. Porcentaje alcanzado de manera general en la aplicación del protocolo Senior Fitness Test, en personas adultas mayores que realizan actividad física

El 74,6 \% de los adultos mayores evaluados presentaron un nivel de aptitud física normal y el 23,2 \% estaba en la categoría de excelente. Finalmente, el 2,2 \% de las personas medidas estuvieron en un nivel de aptitud física deficiente (ver figura 2).

En la figura 3 se muestra que el 86,2\% de los adultos mayores se encontró en valores normales de depresión, el $5,8 \%$ de las personas se ubicó en estado depresivo I y el $8 \%$ restante se mostró como personas depresivas en nivel II. 


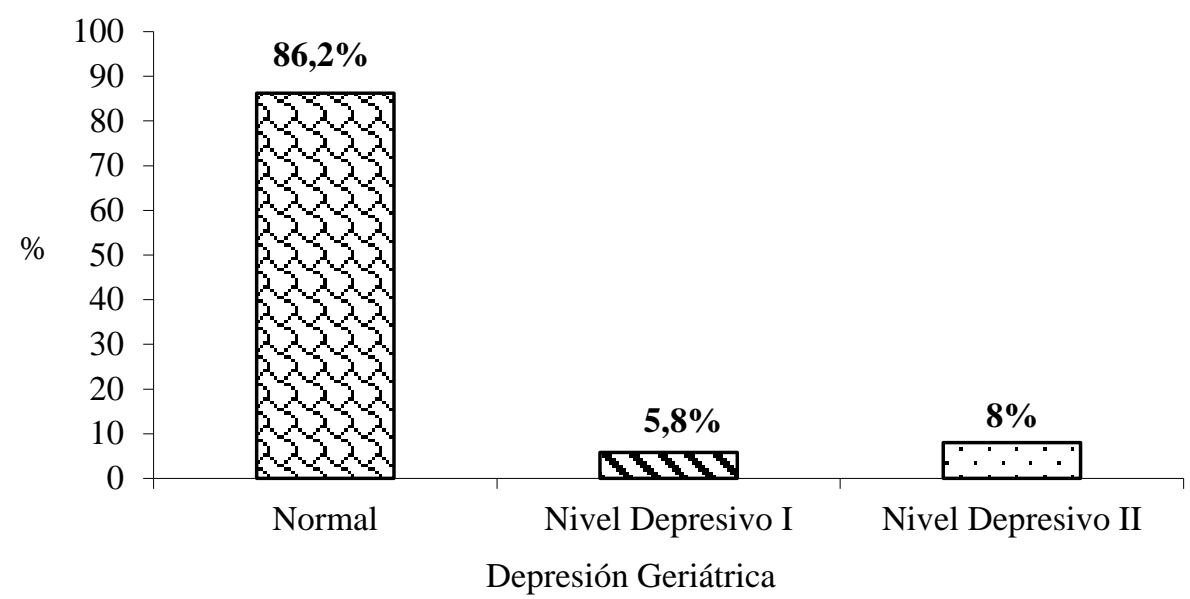

Figura 3. Porcentajes promedio obtenidos en la aplicación de la escala de Depresión Geriátrica de Yesavage en las personas adultas mayores que realizan actividad física

El rango de edad 60-64 años presenta niveles aceptables y el rango de 75 años y más indica la presencia de mayores porcentajes depresivos en las personas analizadas. No se reportan diferencias significativas $(p>0.05)$ entre los rangos de edad (ver figura 3 ).

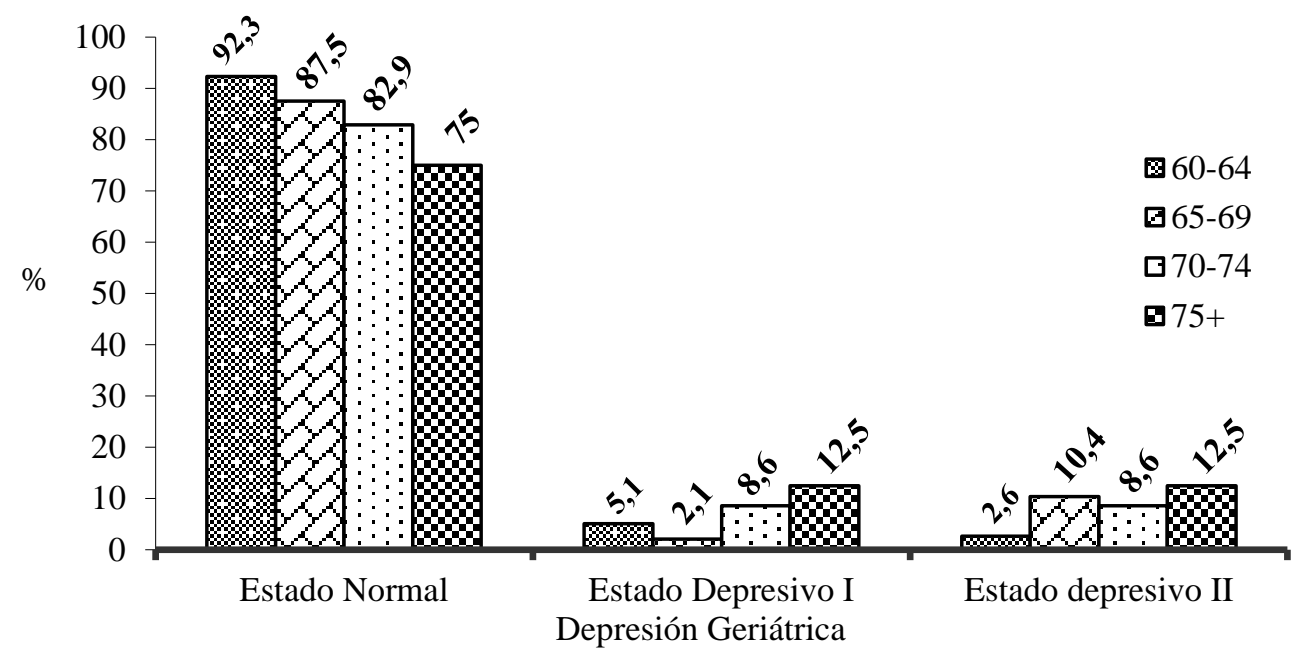

Figura 4. Porcentajes obtenidos mediante el GDS por rango de edad en los estadíos normal, depresión estado I y depresión estado II, en personas adultas mayores que realizan actividad física.

En la figura 5 se presentan las correlaciones entre la escala de depresión geriátrica y las dimensiones del SFT. Se observó que se presentaron asociaciones negativas entre Levantarse y Sentarse de una silla, Flexiones de brazo, Levantarse caminar realizando la figura de un 8 y sentarse $(p<0.05)$ y la prueba de flexión de tronco en silla con la escala de depresión $(p<0.01)$. 


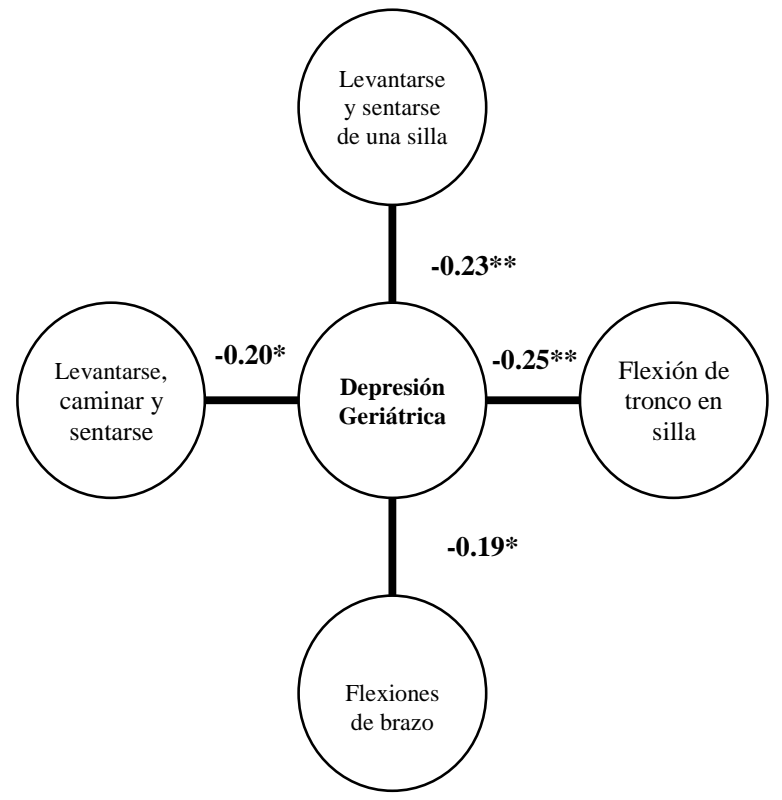

Figura 5. Coeficiente de correlación entre SFT y la DGS de Yesavage, en las personas adultas mayores que realizan actividad física. $* *$ La correlación es significativa al nivel de $p<0.01 *$ La correlación es significativa al nivel de $p<0.05$

\section{Discusión}

La depresión es una enfermedad con proyecciones abrumadoras para el futuro y se relaciona directamente con la condición emocional, la actividad social, la salud y la calidad de vida de las personas adultas mayores (Wada y otros, 2005). En el presente estudio, se encontró que el 86,2 \% de las personas analizadas estaban en un rango normal de depresión. Sin embargo, el 13,8 \% de las personas se ubicó en los niveles depresivos I y II. Estos datos coinciden con los hallazgos encontrados en otros estudios en donde las personas con un mayor nivel aptitud física tuvieron un mejor estado de ánimo (Cáceres, 2004; OPS 2004; Saks, Tiit, Kaarik y Jaanson, 2002; Wada y otros, 2005; Whooley, Stone y Soghikian, 2000).

Por su parte, los resultados de este estudio muestran que a pesar de que no hubo diferencias significativas entre los rangos de edad, se encontró que conforme aumenta la edad los niveles de depresión son mayores. Las personas adultas mayores tienen mayor probabilidad de desarrollar depresión, este hallazgo permite comprender la importancia de realizar una intervención oportuna para que los niveles depresivos no avancen a niveles significativos. En el rango de 60 a 65 años, el 92,3 \% de las personas adultas mayores se ubicó en estado depresivo normal y presentó un 7,7 \% en los niveles depresivos I y II, pero en el rango de edad de 75 años o más la situación cambia a un $75 \%$ de personas en estado normal y un $25 \%$ ubicadas en estados depresivos I y II. Según Hays y otros (1997), los mayores niveles depresivos presentados en las personas adultas mayores pueden ser debido a la pérdida de los seres queridos, a la pérdida paulatina de la capacidad funcional o la dificultad para realizar actividades diarias que antes eran sencillas, lo cual afecta el estado anímico y aumenta los estados depresivos.

Otra posible explicación a este fenómeno lo reportan en varios estudios Wada y otros (2005), Orfila y otros (2006) y Torre y otros (2008), en donde se indica que las personas adultas mayores poseen más enfermedades crónicas no transmisibles (ECNT) como las 
Rodríguez Hernández, M., Araya Ramírez, F., Ureña Bonilla, P., Wadsworth1, D.D. y Solano Mora, L. enfermedades cardiovasculares, el cáncer, la artritis y dolores de la espalda baja en comparación con personas más jóvenes. Este aumento en las ECNT favorece la aparición de síntomas depresivos aproximadamente tres meses después de la aparición de la enfermedad en las personas adultas mayores en donde los niveles de depresión suelen aumentar.

Además, otros factores que contribuyen aumentar los niveles de depresión son la soledad y el cambio de rol diario luego de la jubilación, los cuales podrían afectar negativamente la salud de las personas y los sentimientos depresivos que se hacen más presentes en la etapa adulta mayor (Heun y Hein, 2010; Wada y otros, 2004).

Asimismo, los rasgos depresivos se correlacionaron negativamente con factores directos de la aptitud física. Esto indica que las personas con rasgos depresivos importantes obtuvieron evaluaciones bajas en cuanto a su capacidad física en las dimensiones de flexibilidad, agilidad y equilibrio dinámico, y la funcionalidad de sus piernas. Dicho de otro modo, las personas en buen estado físico manifestaron niveles depresivos bajos, mostrando la relación positiva que tiene la actividad física en el manejo de la depresión en personas adultas mayores. En estudios previos se encontró que las personas con niveles bajos de aptitud física presentaron depresión crónica, que el bajo nivel de actividad física fue predictor de niveles depresivos altos y, además, que al aumentar la actividad física, la depresión disminuyó (Hays y otros 1997; Dunn, Trivedi y O'neal, 2001; Orfila y otros, 2006). Así por ejemplo, las pruebas de flexibilidad, levantarse, caminar y sentarse, y levantarse y sentarse de la silla, correlacionaron negativamente con la depresión.

De acuerdo con los hallazgos presentados por Dunn y otros (2001), hay soporte suficiente para aseverar la relación que existe entre capacidad física funcional y depresión, pues las personas con niveles bajos de actividad física o capacidad física tienen mayores posibilidades de deprimirse; asimismo, las personas que manifestaron niveles depresivos elevados e iniciaron con programas de actividad física incrementaron su capacidad física y disminuyeron los niveles depresivos. De igual modo, Al-Shair y otros (2008) y Torre y otros (2008) encontraron que la depresión estuvo asociada con una pobre capacidad física funcional, además, encontraron que la mejoría en la capacidad física funcional producía efectos positivos sobre la salud, la vitalidad, las actividades de la vida diaria y la depresión entre otros factores.

Este estudio permite interpretar aspectos importantes que tienen que ver con la aptitud física y la depresión en personas adultas mayores que realizan actividad física en grupos organizados. La comprensión de estos permitirá tanto a investigadores como a promotores de programas de actividad física y a las mismas personas adultas mayores desarrollar programas orientados a mejorar la capacidad física funcional y velar por fortalecimiento holístico de esta población. Sin embargo, en posteriores estudios se podrán incluir aspectos como la experiencia previa realizando actividad física para determinar si el tiempo realizando actividad física influye en los niveles depresivos que presentan estas personas. Un diseño de investigación que incluya una intervención y un grupo control servirá para determinar si los cambios en la aptitud física influyen en los niveles depresivos de las personas adultas mayores. 
Por lo anterior, se concluye que los bajos niveles de aptitud física en las personas adultas mayores se relacionaron negativamente con los grados de depresión. Los adultos mayores que mostraron un mayor nivel de aptitud física presentaron niveles depresivos más bajos y los niveles depresivos en estas personas se incrementan conforme avanza la edad.

\section{Referencias Bibliográficas}

Al-Shair, K., Dockry, R., Mallia-Milanes, B., Kolsum, U., Singh, D., \& Vestbo, J. (2008). Depression and its relationship with poor exercise capacity, BODE index and muscle wasting in COPD. ELSEVIER. Journal of Respiratory Medicine, 103, 1572-1579. http://dx.doi.org/10.1016/j.rmed.2008.11.021

Cáceres, R. (2004). Estado de salud, físico y mental de los adultos mayores del área rural de Costa Rica, 2000. Revista Electrónica Población y Salud en Mesoamérica, 1(2), 1-28. http://dx.doi.org/10.15517/psm.v1i2.13931

Chaves, J., Lozano, E., Lara, A. y Velázquez, O. (s.f). La actividad física y el deporte en el adulto mayor, bases fisiológicas. México. Recuperado de http://www.salud.gob.mx/unidades/cdi/documentos/DOCSAL7516.pdf

Dunn, A., Trivedi, M., \& O’Neal, H. (2001). Physical activity dose-response effects on outcomes of depression and anxiety. Medicine \& Science in Sports \& Exercise, 33(6), 587-597. Recuperado de http://journals.lww.com/acsmmsse/Fulltext/2001/06001/Physical_activity_dose_respons e_effects_on.27.aspx

Hays, J., Krishnan, R., George, L., Pieper, C., Flintfd, E., \& Blazera, D. (1997). Psychosocial and physical correlates of chronic depression. Psychiatry Research, 72, 149-159 http://dx.doi.org/10.1016/S0165-1781(97)00105-4

Heun, R., \& Hein, S. (2005). Risk factors of major depression in the elderly. European Psychiatry, 20(3), 199-204. http://dx.doi.org/10.1016/j.eurpsy.2004.09.036

Kurlowicz, L. (2007). La escala de depresión geriátrica (GDS, por sus siglas en inglés). The Hartford Institute for Geriatric Nursing, New York University, College of Nursing, 4. Recuperado de http://consultgerirn.org/uploads/File/trythis/try_this_4_span.pdf

Mather, A., Rodriguez, C., Guthrie, M., McHarg, A., Reid, I., \& McMurdo, M. (2002). Effects of exercise on depressive symptoms in older adults with poorly responsive depressive disorder Randomized controlled trial. The British Journal of Psychiatry, 180. 411-415. http://dx.doi.org/10.1192/bjp.180.5.411

Menchetti, M., Cevenini, N., De Ronchi, D., Quartesan, R., \& Berardi, D. (2006). Depression and frequent attendance in elderly primary care patients. General Hospital Psychiatry, 28(2), 119- 124. http://dx.doi.org/10.1016/j.genhosppsych.2005.10.007 
Mura, G., \& Carta, M. (2013). Physical activity in depressed elderly. A systematic review.

Clinical Practice \& Epidemiology in Mental Health, 9, 125-135.

http://dx.doi.org/10.2174/1745017901309010125

Orfila, F., Ferrer, M., Lamarca, R., Tebe, C., Domingo-Salvany, A., \& Alonso, J. (2006). Gender differences in health-related quality of life among the elderly: The role of objective functional capacity and chronic conditions. Social Science \& Medicine, 63, 2367-2380. http://dx.doi.org/10.1016/j.socscimed.2006.06.017

Organización Panamericana de la Salud. (2004). La salud de las personas adultas mayores en Costa Rica / Organización Panamericana de la Salud, Ministerio de Salud, Consejo Nacional del Adulto Mayor. -- San José, C.R.: OPS 56(16) (Serie Análisis de Situación de Salud. 12. Recuperado de http://bvs.sa.cr/php/situacion/adulto.pdf

Rikli, R., \& Jones, J. (2001). Senior Fitness Test Manual. Human Kinetics. USA

Runcan, P., Hategan, M., Carmen Barbat, C., \& Alexiu, M. (2010). The emergence of depression in the elderly. Procedia Social and Behavioral Sciences, 2(2), 4966-4971. http://dx.doi.org/10.1016/j.sbspro.2010.03.804

Saks, K., Tiit, E., Kaarik, E., \& Jaanson, K. (2002). Depressive symptoms in older Estonians: prevalence and models. Journal of the American Geriatrics Society, 50(6), 1164-1165. http://dx.doi.org/10.1046/j.1532-5415.2002.50280.x

Strawbridge, W., Deleger, S., Roberts, R., \& Kaplan, G. (2002). Physical activity reduces the risk of subsequent depression for older adults. American Journal of Epidemiology, 156(4), 328-334. http://dx.doi.org/10.1093/aje/kwf047

Torre, F., Martín-Corral J., Callejo A., Gómez-Vega C., La Torre S., Esteban, I., Vallejo R. M., \& Arizaga, A. (2008). Calidad de vida relacionada con la salud y estrategias de afrontamiento ante el dolor en pacientes atendidos por una unidad de tratamiento del dolor. Revista Sociedad Española del Dolor, 15(2), 83-93. Recuperado de http://scielo.isciii.es/pdf/dolor/v15n2/original3.pdf

Unsar, S., \& Sut, N. (2010), Depression and health status in elderly hospitalized patients with chronic illness. Archives of Gerontology and Geriatrics, 50(1), 6-10. http://dx.doi.org/10.1016/j.archger.2008.12.011

Wada, T., Ishine, M., Sakagami, T., Kita, T., Okumiya, K., Mizuno, K., Rambo, T.A., \& Matsubayashi, K. (2005). Depression, activities of daily living, and quality of life of community-dwelling elderly in three Asian countries: Indonesia, Vietnam, and Japan. Archives of Gerontology and Geriatrics, 41(3). 271-280. http://dx.doi.org/10.1016/j.archger.2005.03.003

Wada, T., Ishine, M., Sakagami T., Okumiya, K., Fujisawa, M., Murakami, S., Otsuka, K., Yano, S., Kita, T., \& Matsubayashi K. (2004). Depression in Japanese communitydwelling elderly - prevalence and association with ADL and QOL. Archives of Gerontology and Geriatrics, 39, 15-23. http://dx.doi.org/10.1016/j.archger.2003.12.003

Revista MHSalud® (ISSN: 1659-097X) Vol. x. No. x. Setiembre-Enero, 2014 
Whooley, M. A., Stone, B., \& Soghikian, K. (2000). Randomized trial of case-finding for depression in elderly primary care patients. Journal General Internal Medicine, 15(5), 293-300. http://dx.doi.org/10.1046/j.1525-1497.2000.04319.x

Recepción: 5 de junio del 2014

Corrección: 11 de agosto del 2014

Aceptación: 18 de agosto del 2014

Publicación: 31 de agosto del 2014 